\title{
Generalized Riccati Solution and Pinning Control of Complex Stochastic Networks
}

\author{
Randa Herzallah ${ }^{\mathrm{a}, *}$, \\ ${ }^{a}$ Non-linearinty and Complexity Research Group, Aston University, Birmingham, \\ United Kingdom \\ E-mail: r.herzallah@aston.ac.uk
}

\section{Abstract}

This paper considers the global synchronization of a stochastic version of coupled map lattices networks through an innovative stochastic adaptive linear quadratic pinning control methodology. In a stochastic network, each state receives only noisy measurement of its neighbors states. For such networks we derive a generalized Riccati solution that quantifies and incorporates uncertainty of the forward dynamics and inverse controller in the derivation of the stochastic optimal control law. The generalized Riccati solution is derived using the Liapunov approach. A probabilistic approximation type algorithms are employed to estimate the conditional distributions of the state and inverse controller from historical data and quantifying models uncertainty. The theoretical derivation is complemented by its validation on a set of representative examples.

\section{Introduction}

Current and future emergent systems in nature are complex systems which can be viewed as complex networks consisting of a large number of elements interacting with each other. Numerous fields of science and engineering have witnessed new research and interest in regulating complex networks including energy efficient controller of wireless sensor networks, control mechanisms of cell cycles [13], and the control of the respiratory rhythm [21]. Pinning control has been proposed in the literature to control lattices and complex networks of coupled dynamical systems [23,27-29]. In pinning control, control actions are applied on a limited subset of the dynamical system placed at a fraction of the network nodes. The effect of these actions is then propagated to the rest of the network through the coupling among the network nodes.

Corresponding author. E-mail: r.herzallah@aston.ac.uk.
Previous pinning control methodologies are based mainly on linear feedback control theory. In [13], linear control theory was proposed to develop an improved localized control scheme for spatiotemporally chaotic systems which was then applied to a coupled map lattice (CML). The result that a network under a typical framework can realize synchronization subject to any linear feedback pinning scheme by using adaptive tuning of the coupling strength was proved in [31]. The stabilization problem of complex dynamical networks with general coupling topology by pinning a small fraction of nodes with local negative feedback controllers was discussed in [30]. However linear feedback control theory relies on the availability of a model that characterizes the dynamics of the system to be controlled. Thus the model of the plant whether obtained a priori or during control is a key feature to pinning control design. Previous work on pinning control on the other hand has always used the exact model of the network to calculate the various control actions. This represents the control solution by assuming that the system is certainty equivalent $[2,7,9,12]$ 
even when this is not the case. Therefore, if the dynamics of the actual network are unknown a priori the certainty equivalent control is likely to perform unsatisfactorily $[8,9,16,17]$. Another assumption of previous work on pinning control is that the networks could be chaotic but not stochastic. Stochastic networks with noise components affecting the overall dynamics of the network have not been considered. This assumption is rather restrictive since noise plays a significant role in all real world complex systems.

This paper is concerned with the problem of uncertainty propagation through a network of complex dynamical systems by exploiting a new control methodology that takes randomness and functional uncertainties into consideration. We consider a stochastic linearized version of complex coupled map lattice Networks with fixed structure and coupling strength described by the following linear state space model:

$$
\begin{aligned}
\mathbf{x}_{\mathrm{t}+1} & =\mathbf{f}\left(\mathbf{x}_{\mathrm{t}}, \mathbf{u}_{\mathrm{t}}^{\mathrm{op}}, \tilde{\eta}_{\mathrm{t}+1}\right), \\
& =\tilde{\mathrm{G}} \mathbf{x}_{\mathrm{t}}+\tilde{\mathrm{H}} \mathbf{u}_{\mathrm{t}}^{\text {op }}+\tilde{\eta}_{\mathrm{t}+1}
\end{aligned}
$$

where $\tilde{G}$ and $\tilde{H}$ are $n \times n$ and $n \times m$ matrices respectively, $\mathbf{u}^{\text {op }} \in R^{m}$ is the input vector, $\mathbf{x} \in R^{n}$ is the state vector and $\tilde{\eta}$ is random independent noise with zero mean and covariance $\tilde{\Sigma}$. During the past two decades, a number of theories have been developed for linear systems given in (1) that is related to the problem of identification [5], control [32], model reduction and filtering $[1,14]$. In optimal linear quadratic (LQ) control theory, the Ricatti equation approach has been widely used to provide an optimal feedback control for deterministic and stochastic control systems $[3,25,26]$. The optimal control of linear systems with uncertain parameters and quadratic cost functions has then been formulated in the context of guaranteed cost control $[4,6]$. For these uncertain systems, linear constant gain feedback controllers which depend on the solution of generalized Ricatti equations are designed. Generalized Ricatti equations are Ricatti equations with additional terms that take into account the effect of uncertainties in the control design. Various forms of the generalized Riccati equations appeared in the literature [22, 24]. Each form corresponds to a different uncertainty description and to a specific choice of upper bounding functions.

The aim here is to estimate the forward dynamics of a linearized stochastic class of CML network specified by the general form (1) as function of the connectivity strength and positions of control laws in the network and to estimate the uncertainty of these es- timated models. Models uncertainty are estimated by on-line computation of the conditional probability distributions of the forward model and the inverse controller [18]. A generalized steady state Ricatti equation that minimizes a predefined quadratic cost function is then derived using the Liapunov approach. The effect of functional uncertainties on the stability of complex networks will also be investigated. Compared with existing results on the topic, this paper has the following distinct features: A probabilistic rather than deterministic control algorithm is derived via the Liapunov approach, The derived control algorithm takes into considerations models uncertainty in forward and inverse dynamics, and the conditional distribution rather than deterministic models of the network dynamics are estimated on-line.

\section{Problem Formulation and Preliminaries}

Consider the coupled map lattice consisting of L lattice nodes with periodic boundary conditions $[10,13$, 20], and whose dynamics are driven by stochastic components as follows,

$$
\begin{aligned}
z_{\mathrm{t}+1}^{\mathrm{i}} & =\mathrm{F}\left(z_{\mathrm{t}}^{\mathrm{i}-1}, z_{\mathrm{t}}^{\mathrm{i}}, z_{\mathrm{t}}^{\mathrm{i}+1}\right) \\
& =\mathrm{f}\left[(1-2 \epsilon) z_{\mathrm{t}}^{\mathrm{i}}+\epsilon\left(z_{\mathrm{t}}^{i-1}+z_{\mathrm{t}}^{i+1}\right)\right] \\
& +\tilde{\mathrm{\kappa}}_{\mathrm{t}+1}^{\mathrm{i}},
\end{aligned}
$$

where $i=1,2, \ldots, L$ are the lattice sites, $L$ is the system size, $\tilde{\kappa}_{t+1}^{i}$ is an additive noise signal assumed to have zero mean Gaussian distribution of covariance $\tilde{\Gamma}$, and the periodic boundary conditions are given by $z_{\mathrm{t}}^{\mathrm{i}+\mathrm{L}}=z_{\mathrm{t}}^{\mathrm{i}}$. The local map $\mathrm{f}(z)$ is defined to be a nonlinear function of the following form

$$
f(z)=a z(1-z)
$$

This coupled map lattice exhibits chaotic characteristics in the regime $3.57<a \leq 4.0$ and has a homogeneous steady state $z^{\star}=1-1 / \mathrm{a}$. The goal in pinning control is then to stabilize the homogeneous state by applying $M$ periodically control actions placed at sites $\left\{i_{1}, \ldots, i_{M}\right\}$ in the following way

$$
\begin{aligned}
z_{\mathrm{t}+1}^{\mathrm{i}} & =\mathrm{F}\left(z_{\mathrm{t}}^{\mathrm{i}-1}, z_{\mathrm{t}}^{\mathrm{i}}, z_{\mathrm{t}}^{\mathrm{i}+1}\right)+\sum_{\mathrm{m}=1}^{\mathrm{M}} \delta\left(i-i_{\mathrm{m}}\right) \mathrm{u}_{\mathrm{t}}^{\mathrm{m}} \\
& +\tilde{\kappa}_{\mathrm{t}+1}^{\mathrm{i}},
\end{aligned}
$$

where $u_{m}$ is the control action applied at site $m$. To calculate the feedback control actions, the theory 
of linear quadratic control is used [13]. For that purpose Equation (4) is linearized about the homogeneous steady state $\mathbf{z}_{\mathrm{t}}=\left(z_{1}^{\star}, \ldots, z_{\mathrm{L}}^{\star}\right)$ to become as follows

$$
\mathbf{x}_{\mathrm{t}+1}=\tilde{\mathrm{G}} \mathbf{x}_{\mathrm{t}}+\tilde{\mathrm{H}} \mathbf{u}_{\mathrm{t}}+\tilde{\mathrm{K}}_{\mathrm{t}+1},
$$

in which $\mathbf{x}=\mathbf{z}-\mathbf{z}^{\star}$ represents the state vector, the $\mathrm{L} \times \mathrm{L}$ Jacobian matrix $\tilde{\mathrm{G}}$, is given by

$$
\tilde{\mathrm{G}}=\alpha\left[\begin{array}{ccccc}
1-2 \epsilon & \epsilon & 0 & \ldots & \epsilon \\
\epsilon & 1-2 \epsilon & \epsilon & \ldots & 0 \\
0 & \epsilon & 1-2 \epsilon & \ldots & 0 \\
\vdots & \vdots & \vdots & \ddots & \vdots \\
\epsilon & 0 & 0 & \ldots & 1-2 \epsilon
\end{array}\right],(6)
$$

where $\alpha=\left.\frac{\partial f(z)}{\partial z}\right|_{z=z^{\star}}$, and $\tilde{H}$ is an $L \times M$ control matrix with $\tilde{H}_{i j}=\sum_{m} \delta(j-m) \delta\left(i-i_{m}\right)$. Thus, the pinning controlled network can be described by

$$
\mathbf{x}_{\mathrm{t}+1}=(\tilde{\mathrm{G}}-\tilde{\mathrm{H} K}) \mathbf{x}_{\mathrm{t}},
$$

in which the control action is defined to be of the following form,

$$
\mathbf{u}_{\mathrm{t}}=-\mathrm{K} \mathbf{x}_{\mathrm{t}} .
$$

Here $\mathrm{K}$ is the feedback gain that is evaluated such that the following cost functional is minimized

$$
J=\frac{1}{2} \sum_{\mathrm{t}=0}^{\infty}\left[\mathbf{x}_{\mathrm{t}}^{*} \mathrm{Q} \mathbf{x}_{\mathrm{t}}+\mathbf{u}_{\mathrm{t}}^{*} \mathrm{R} \mathbf{u}_{\mathrm{t}}\right]
$$

where $Q$ and $R$ are positive definite matrices. If the state matrix, $\tilde{\mathrm{G}}$ and control matrix, $\tilde{\mathrm{H}}$ were known and the network was noiseless then the feedback gain that minimizes equation (9) is shown to be given by

$$
K=\left(R+\tilde{H}^{*} P \tilde{H}\right)^{-1} \tilde{H}^{*} P \tilde{G}
$$

where $\mathrm{P}$ is the Riccati equation which can be calculated as follows

$$
P=Q+\tilde{G}^{*} P \tilde{G}-\tilde{G}^{*} P \tilde{H}\left[R+\tilde{H}^{*} P \tilde{H}\right]^{-1} \tilde{H}^{*} P \tilde{G} .
$$

Since the coupled map lattice has parity symmetry, the eigenvalues of its Jacobian (6) are doubly degenerate. Therefore, the minimal number of controllers that yield a controllable system in this case is two [13]. The feedback gain of the two pinning sites is then computed from (10), which yields the optimal control actions of the deterministic known systems. It also yields optimal control actions if the additive noise of the system is input independent. This framework, which considers exact known systems, has always been assumed in the pinning control literature. It is equivalent to the well known certainty equivalent assumption in conventional control theory.

The pinning control problem for systems with unknown state and control parameters however, should be formulated in an adaptive control framework which is shown to have functional uncertainty. The theory of linear quadratic control will be extended in this paper such that models uncertainty are taken into consideration in the feedback control. The generalized Riccati solution will then be derived using the Liapunov approach, hence a stabilizable stochastic control law is obtained. The stochastic feedback control will be introduced soon, but first we give a brief discussion on estimation of the conditional response of the coupled map lattice network.

\section{Conditional Distribution of Linearized Coupled Map Lattice}

In adaptive and optimal control, researchers usually estimate the parameters of the forward dynamics of the system and use these estimates to represent the unknown parameters of the system model. This implicitly implies that an accurate identification for the parameters of the system model can be obtained. However, in most control applications it is practically impossible to obtain an accurate mathematical model of the dynamics. Therefore, researchers have recently considered modeling the conditional distributions of the stochastic systems rather than relying on the single estimate of their parameters [9, 17-19].

To estimate the conditional distribution of the linearized CML, a generalized linear model is optimized in this paper such that its output approximates the conditional expectation of the state. According to Theorem 4.2.1 in [11], the conditional expectation of the system state defined in (5) can be estimated using a generalized linear network such that the mean square error between the measured state from the CML, $\mathbf{x}_{\mathrm{t}+1}$ and the estimated state values, $\hat{\mathbf{x}}_{\mathrm{t}+1}$ is minimized. Once the output of the generalized network has been optimized, the stochastic model of the coupled map lattice network defined in Equation (5) can then be described as follows:

$$
\begin{aligned}
\mathbf{x}_{\mathrm{t}+1} & =\widehat{\mathbf{x}}_{\mathrm{t}+1}+\eta_{\mathrm{t}+1}, \\
& =\mathrm{G} \mathbf{x}_{\mathrm{t}}+\mathrm{H} \mathbf{u}_{\mathrm{t}}^{\text {op }}+\eta_{\mathrm{t}+1},
\end{aligned}
$$


where $\hat{\mathbf{x}}_{\mathbf{t}+1}=\hat{\mathbf{f}}\left(\mathbf{x}_{\mathbf{t}}, \mathbf{u}_{\mathbf{t}}, \theta\right)$ is the parameterized linear model that provides prediction for the expected values of the state, $\mathrm{G}$, and $\mathrm{H}$ are the estimated state and control matrices respectively, and $\eta_{t+1}$ represents an input and state dependent zero mean random noise.

To estimate the probability density function of the state, another generalized linear network, which has the same structure and same inputs as that of the predicted value of the state model, is then used to provide an estimate for the state and control dependent noise $\eta_{t+1}$ as follows,

$$
\eta_{t+1}=D \mathbf{x}_{t}+E \mathbf{u}_{t}^{o p}
$$

where here the elements of the matrices D and E are adapted such that they have zero mean and a covariance matrix $\left\langle\eta_{t+1} \eta_{t+1} *>\right.$. Further details about estimating the conditional distributions of the systems dynamics can be found in [15].

\section{Stochastic Feedback Control}

The conventional solution of the linear quadratic control discussed in Section 3 assumes that exact models of the coupled map lattice network are available. However, since the models of most real world applications are usually unknown, this assumption is generally not realistic in practice, therefore an appropriate method for estimating the network models should be used. This is known to have functional uncertainty which should be considered when deriving the optimal control law. Hence, for the derivation of the stochastic feedback control algorithm proposed in this paper, this assumption is relaxed by estimating the conditional distribution of the predicted states of the CML. The estimation of the conditional distribution rather than the deterministic model of the system dynamics allows the quantification and incorporation of models uncertainty in the derivation of the optimal control law.

For the discrete time stochastic model of the CML defined in (5), the expected value of the cost functional defined in Equation (9) should be minimized,

$$
\mathrm{J}\left(\mathrm{t}_{\mathrm{o}}, \mathbf{x}_{\mathrm{t}_{\mathrm{o}}} ; \mathbf{u}_{\mathrm{a}}\right)=\mathrm{E}\left(\frac{1}{2} \sum_{\mathrm{t}=\mathrm{t}_{\mathrm{o}}}^{\infty}\left[\mathbf{x}_{\mathrm{t}}^{*} \mathrm{Q} \mathbf{x}_{\mathrm{t}}+\mathbf{u}_{\mathrm{t}}^{*} \mathrm{R} \mathbf{u}_{\mathrm{t}}\right]\right)
$$

where we have now made the dependency of the cost function on the initial time $t_{0}$, the initial state $\mathbf{x}_{t_{0}}$ and admissible control values $\mathbf{u}_{\mathrm{a}}$ explicit. The set of all admissible control is denoted by $\mathrm{U}_{\mathrm{a}}$. The solution $\mathbf{x}_{\mathrm{a}}$ of the system (5) is called the response of the admissible control, and the pair $\left(\mathbf{u}_{\mathrm{a}}, \mathbf{x}_{\mathrm{a}}\right)$ is called an admissible pair. The objective of the optimal control problem is then to minimize the cost function (14) for a given $\left(\mathrm{t}_{\mathrm{o}}, \mathbf{x}_{\mathrm{t}_{\mathrm{o}}}\right)$, over all $\mathbf{u}_{\mathrm{a}} \in \mathrm{U}_{\mathrm{a}}$ subject to (5).

Under the assumption of exact model parameters and the positive definiteness of the matrices $Q$ and $R$, the minimization of (14) subject to the linear model of equation (5) reduces to the standard linear quadratic control problem discussed in Section 3.

However, when models' parameters are unknown the linear quadratic control problem should be formulated in an adaptive control framework. Here the conditional distribution of the linear systems (5) should be estimated as discussed in Section 4. The minimization of (14) is then subjected to the constraint equations specified by (12) and (13), repeated here

$$
\begin{aligned}
& \mathbf{x}_{\mathrm{t}+1}=\mathrm{G} \mathbf{x}_{\mathrm{t}}+\mathrm{H} \mathbf{u}_{\mathrm{t}}^{\mathrm{op}}+\eta_{\mathrm{t}+1}, \\
& \eta_{\mathrm{t}+1}=\mathrm{D} \mathbf{x}_{\mathrm{t}}+\mathrm{E}_{\mathrm{t}}^{\mathrm{op}}
\end{aligned}
$$

This leads to the stochastic optimal feedback control law specified in the following theorem.

Theorem 1. The feedback control law minimizing performance index of (14) subject to the system model of (15) and the error model of (16)

$$
\mathbf{u}^{\mathrm{op}}=-\mathrm{K}_{\mathrm{t}} \mathbf{x}_{\mathrm{t}}
$$

with

$$
\begin{aligned}
\mathrm{K}_{\mathrm{t}} & =\left[\mathrm{R}+\mathrm{H}^{*} \mathrm{PH}+<\mathrm{E}^{*} \mathrm{PE}>\right]^{-1} \\
& \times\left[\mathrm{H}^{*} \mathrm{PG}+<\mathrm{E}^{*} \mathrm{PD}>\right],
\end{aligned}
$$

is a stabilizing control law and

$$
\mathrm{J}\left[\mathbf{x}_{\mathrm{t}}\right]=\mathbf{x}_{\mathrm{t}}^{*} \mathrm{P} \mathbf{x}_{\mathrm{t}},
$$

with

$$
\begin{aligned}
\mathrm{P} & =\mathrm{Q}-\left[\mathrm{G}^{*} \mathrm{PH}+<\mathrm{D}^{*} \mathrm{PE}>\right]\left[\mathrm{R}+\mathrm{H}^{*} \mathrm{PH}+<\mathrm{E}^{*} \mathrm{PE}>\right]^{-1} \\
& \times\left[\mathrm{H}^{*} \mathrm{PG}+<\mathrm{E}^{*} \mathrm{PD}>\right]+\mathrm{G}^{*} \mathrm{PG}+<\mathrm{D}^{*} \mathrm{PD}>
\end{aligned}
$$

is the quadratic cost function.

Proof. The proof of this theorem is based on the Liapunov method. It is given in the Appendix.

A generalized linear model is also proposed in this paper to estimate the optimal control laws as calculated from (17). Once this model is trained and optimized such that it provides prediction for the control law, it can be used at any time to predict control sig- 
nals as long as the system under consideration is time invariant. Thus the stochastic optimal control law (17) is used as the target for the generalized linear model of the inverse controller. The output of this generalized linear model is the estimated control law, $\mathbf{u}_{\mathrm{t}}$ and the stochastic model of the controller can be formulated as,

$$
\begin{aligned}
\mathbf{u}_{\mathrm{t}}^{\mathrm{op}} & =\mathbf{u}_{\mathrm{t}}+e_{\mathrm{t}}, \\
& =A \mathbf{x}_{\mathrm{t}}+e_{\mathrm{t}} .
\end{aligned}
$$

The probability density function of the inverse controller, is then estimated following the same method as that of the forward model. Hence another generalized linear network is used to estimate the control error, $e_{t}$ as follows,

$$
e_{\mathrm{t}}=\mathrm{B} \mathbf{x}_{\mathrm{t}}
$$

where here the elements of the matrix B are adapted such that they have zero mean and covariance matrix $<e_{\mathrm{t}} e_{\mathrm{t}}^{*}>$.

\section{Numerical Results}

In this section, by following the two control approaches discussed above, we give numerical evidence of the advantages of incorporating knowledge of uncertainty when deriving the optimal control for complex systems characterized by functional uncertainty.

\subsection{Non Chaotic Map Lattice}

The example considered here is for the logistic coupled map lattice, $f(z)=a z(1-z)$ in its non-chaotic regime with $a=3.0, \epsilon=0.33$ and $L=5$. The positive weighting matrices of the state and control are taken to be $\mathrm{Q}=\mathrm{I}_{5 \times 5}$ and $\mathrm{R}=\mathrm{I}_{2 \times 2}$. The two control actions are placed next to each other at the sides of the lattice. Hence the equation of the coupled map lattice becomes:

$$
\mathbf{x}_{\mathrm{t}+1}=\tilde{\mathrm{G}} \mathbf{x}_{\mathrm{t}}+\tilde{\mathrm{H}} \mathbf{u}_{\mathrm{t}}+\tilde{\mathrm{K}}_{\mathrm{t}+1},
$$

where

$$
\tilde{\mathrm{G}}=\left[\begin{array}{ccccc}
-0.34 & -0.33 & 0 & 0 & -0.33 \\
-0.33 & -0.34 & -0.33 & 0 & 0 \\
0 & -0.33 & -0.34 & -0.33 & 0 \\
0 & 0 & -0.33 & -0.34 & -0.33 \\
-0.33 & 0 & 0 & -0.33 & -0.34
\end{array}\right],
$$

$$
\tilde{H}=\left[\begin{array}{ll}
1 & 0 \\
0 & 0 \\
0 & 0 \\
0 & 0 \\
0 & 1
\end{array}\right] \quad E\left[\kappa^{t+1}\left(\kappa^{t+1}\right)^{T}\right]=0.01 I_{5 \times 5} .
$$

For the two control methods: conventional and stochastic optimal control, the network models are assumed to be unknown. They are estimated on line using generalized linear models as discussed in Section 4, concurrently with the calculation of the optimal feedback control. For the stochastic optimal control method, uncertainties represented by the errors in the forward and inverse dynamics are also estimated on line using another generalized linear models as described by Equations (13) and (22) respectively. Moreover, the initial values of the state is taken to be $\mathbf{x}(0)=10^{-14}$ in both of the control methods. Figure 1 provides a comparison between the synchronized state values and control efforts as obtained from the conventional controller (8) and the stochastic controller (17).

The same experiment above is repeated for larger size of coupled map lattice with 10 sites, i.e $\mathrm{L}=10$. The control results are shown in Figure 2. The two figures show that the performance of the stochastic feedback control is superior to its counterpart of conventional feedback control for networks characterized by functional uncertainty. The conventional controller initially responds crudely with large transient overshoot reflecting the fact that models of the network have not converged to the true network dynamics yet. This is expected since no knowledge of uncertainty is taken into consideration in the optimal control law. However, since the stochastic feedback controller knows that the network models have not converged during the initial period of control it reacts more cautiously with significant reduction in the overshoot during that period. Figure 1.e and 2.e show the estimated and actual values of $\epsilon \alpha$ where it can be seen that the estimated value converges to the actual value after a few time steps.

Comparing Figures 1 and 2 it can be noted that as the complexity of the network which is represented by the size of the lattice increases, the time required to synchronize the state increases. This can be referred to 


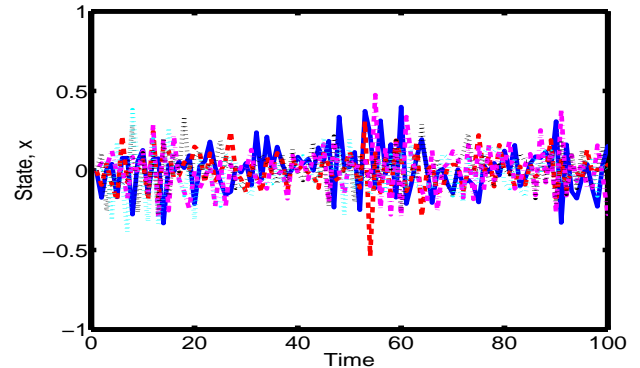

(a)

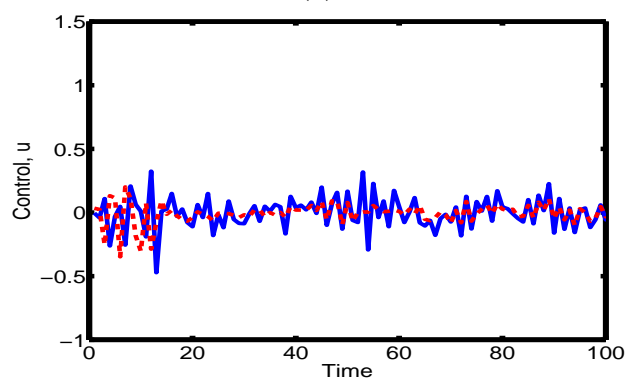

(b)

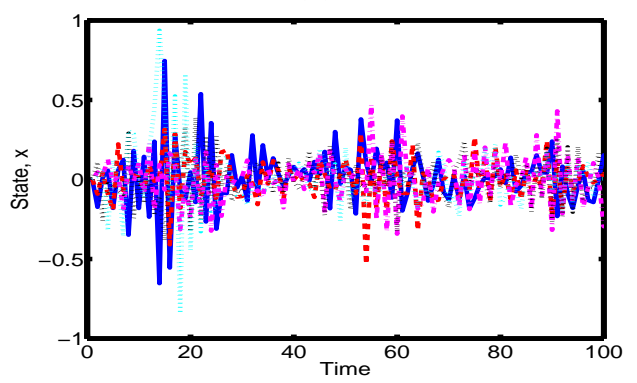

(c)

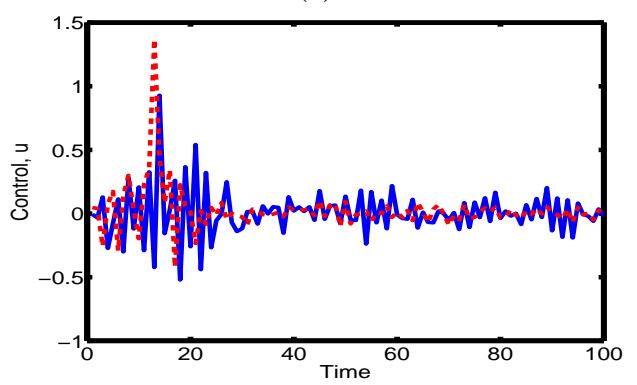

(d)

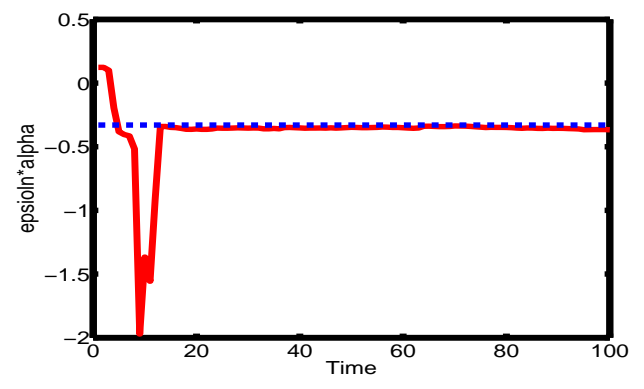

(e)

Fig. 1. States and control efforts of a non chaotic coupled map lattice with, $\mathrm{L}=5, \mathrm{a}=3$, and $\epsilon=0.33$ : (a) States as a result of the stochastic linear quadratic controller. (b) Control efforts from the stochastic linear quadratic controller. (c) States as a result of the conventional linear quadratic controller. (d) Control efforts from the conventional linear quadratic controller. (e) Estimated and actual values of $\epsilon \alpha$. the following two reasons: firstly, as the size of the network increases the complexity of the network model increases so the estimated model takes longer time to converge to the true dynamics of the network which means larger uncertainty as well. Secondly, since only two pinning sites are assumed in the control of the network, the feedback control efforts affect those sites far away from the pinning sites only indirectly through the coupling to the neighbors. This means that networks with higher coupling strength would synchronize quicker.

Our ability to locally control arbitrarily large systems with a specific number of pinning sites is limited. This ability is limited further due to the existence of functional uncertainty. Therefore, further experiments have been conducted to analyze the effect of functional uncertainty on systems stability using the conventional and stochastic control methods. It has been found that the maximal length of the system that can actually be stabilized by the conventional linear quadratic control method is 13 . For the stochastic linear quadratic control method, however, this length is found to be 14 . This is also expected, due to the ignorance of the conventional linear quadratic control to knowledge of uncertainty.

\subsection{Chaotic Map Lattice}

Generally chaotic systems are more difficult to synchronize than non-chaotic systems. For the coupled map lattice defined in Equation (5) chaos can be obtained when $3.5<a \leq 4$. In this section we show the control result in this chaotic regime by taking $a=4$, $\mathrm{L}=6$, and $\epsilon=0.25$.

Figure 3 confirms the result obtained for the nonchaotic map lattice. The control result, for the chaotic map lattice, of the stochastic feedback control is better than that of conventional feedback control, since knowledge of uncertainty is taken into consideration in the former control method. Similarly here for the chaotic map lattice we numerically estimate the maximal length of the system that can be stabilized by conventional and stochastic linear quadratic control methods which are found to be 12 and 13 respectively. This means that our ability to control larger systems with two pinning sites is improved when knowledge of uncertainty is taken into consideration. 


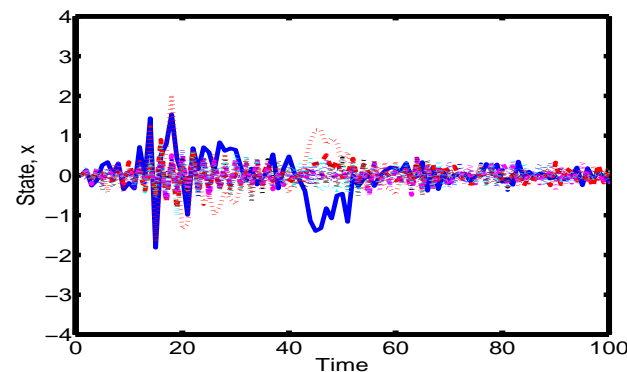

(a)

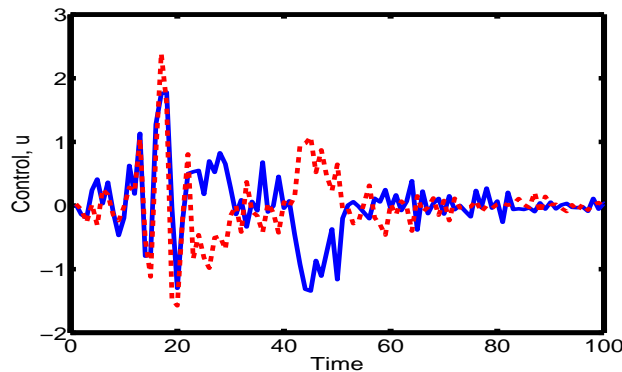

(b)

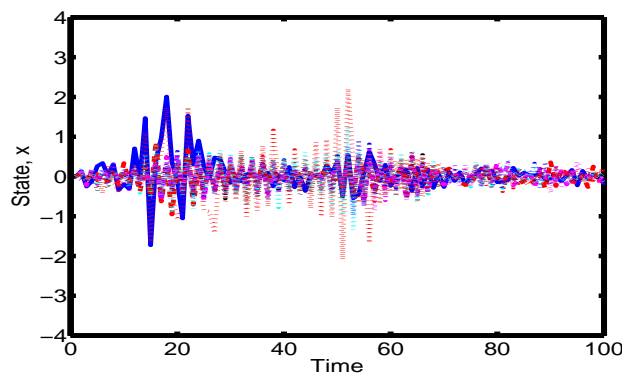

(c)

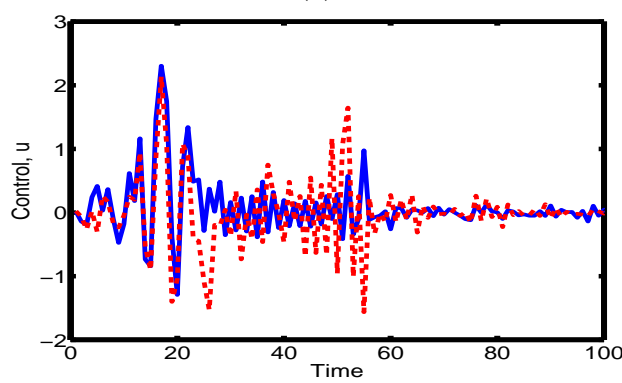

(d)

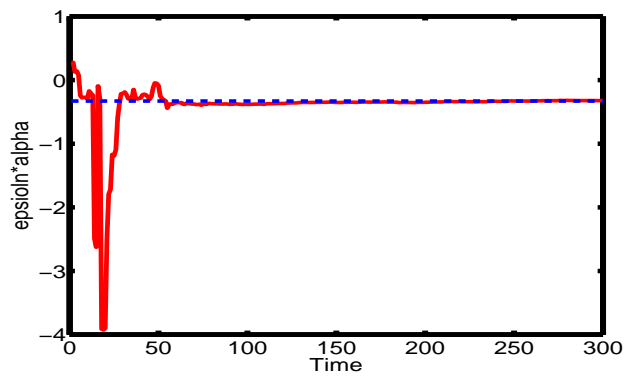

(e)

Fig. 2. States and control efforts of a non chaotic coupled map lattice with, $\mathrm{L}=10, \mathrm{a}=3$, and $\epsilon=0.33$ : (a) States as a result of the stochastic linear quadratic controller. (b) Control efforts from the stochastic linear quadratic controller. (c) States as a result of the conventional linear quadratic controller. (d) Control efforts from the conventional linear quadratic controller. (e) Estimated and actual values of $\epsilon \alpha$.

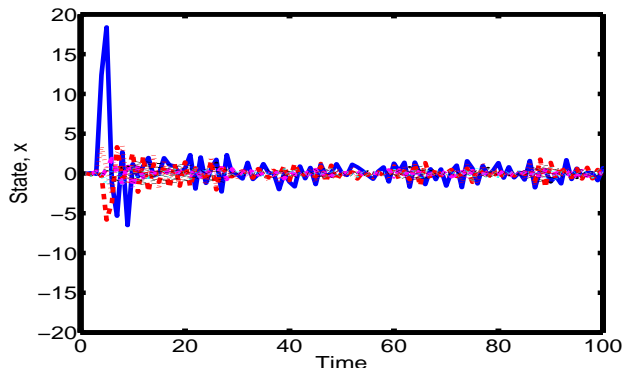

(a)

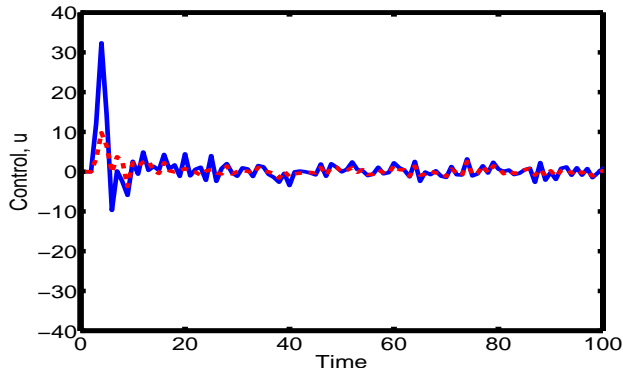

(b)

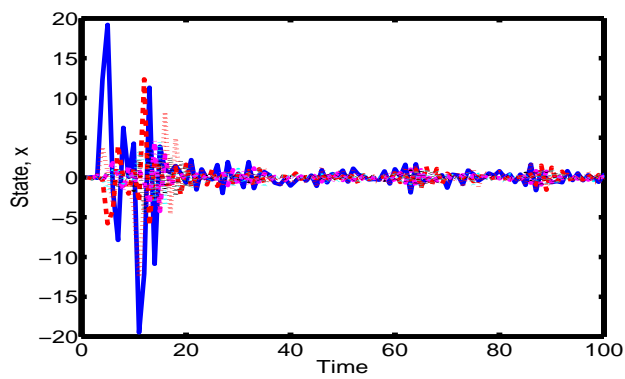

(c)

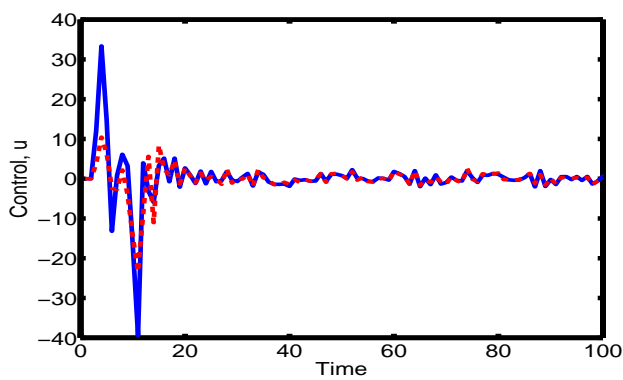

(d)

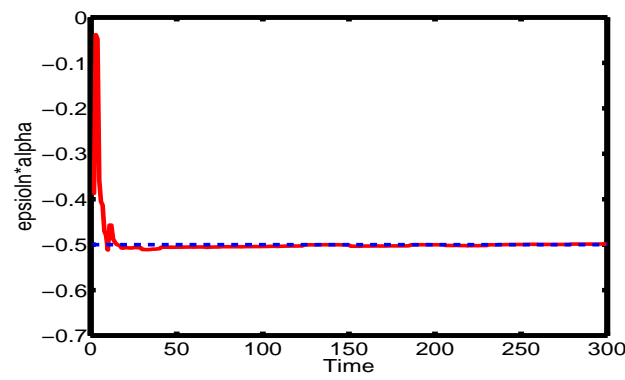

(e)

Fig. 3. States and control efforts of a chaotic coupled map lattice with, $\mathrm{L}=6, \mathrm{a}=4$, and $\epsilon=0.25$ : (a) States as a result of the stochastic linear quadratic controller. (b) Control efforts from the stochastic linear quadratic controller. (c) States as a result of the conventional linear quadratic controller. (d) Control efforts from the conventional linear quadratic controller. (e) Estimated and actual values of $\epsilon \alpha$. 


\section{Conclusion}

In this paper, a new stochastic control algorithm that takes models uncertainty into consideration has been described for a class of complex coupled map lattice with spatiotemporal chaos network that is stochastic as well as chaotic. Since the network is driven by a stochastic random input, the linear quadratic control method is used with the conditional stochastic distributions of the network dynamics rather than deterministic models. The linear quadratic solution is then obtained via the Liapunov approach and a generalized Riccati equation is derived. The derived generalized Ricatti equation is rather now dependent on the estimated models uncertainties. The conventional Riccati solution can be regarded as a special case of the derived generalized Riccati solution by assuming certainty equivalence principle.

Simulation results of the stochastic coupled map lattice have shown that the stochastic controller reacts more cautiously when the models of the network are not converged to the true dynamics, therefore exhibiting less transient overshoots. Moreover, the largest length of the system that can actually be stabilized by stochastic linear quadratic control is shown to be larger than that of conventional linear quadratic control.

\section{Appendix}

Given the assumptions that $\mathrm{Q}$ and $\mathrm{R}$ are positive definite or positive semi-definite Hermitian matrices then a Liapunov function exists that is positive definite and whose derivative is negative definite. For the uncertain quadratic optimal control problem, the following Liapunov function is set,

$$
\mathrm{V}\left(\mathbf{x}_{\mathrm{t}}\right)=\mathbf{x}_{\mathrm{t}}^{*} \mathrm{P} \mathbf{x}_{\mathrm{t}} .
$$

The derivative of the Liapunov function is then given by,

$$
\triangle \mathrm{V}(\mathbf{x}(\mathrm{t}))=<\mathbf{x}_{\mathrm{t}+1}^{*} P \mathbf{x}_{\mathrm{t}+1}>-<\mathbf{x}_{\mathrm{t}}^{*} \mathrm{P} \mathbf{x}_{\mathrm{t}}>
$$

Substituting (12) into (25) yields,

$$
\begin{gathered}
\triangle \mathrm{V}(\mathbf{x}(\mathrm{t}))=<\left[\mathrm{G} \mathbf{x}_{\mathrm{t}}+\mathrm{H} \mathbf{u}_{\mathrm{t}}^{\text {op }}+\eta_{\mathrm{t}+1}\right]^{*} \mathrm{P} \\
\times\left[G \mathbf{x}_{\mathrm{t}}+\mathrm{H} \mathbf{u}_{\mathrm{t}}^{o p}+\eta_{\mathrm{t}+1}\right]>-<\mathbf{x}_{\mathrm{t}}^{*} \mathrm{P} \mathbf{x}_{\mathrm{t}}> \\
=<\mathbf{x}_{\mathrm{t}}^{*} \mathrm{G}^{*} \mathrm{PG} \mathbf{x}_{\mathrm{t}}+\mathbf{x}_{\mathrm{t}}^{*} \mathrm{G}^{*} \mathrm{PH} \mathbf{u}_{\mathrm{t}}^{\text {op }}+\mathbf{u}_{\mathrm{t}}^{* \mathrm{op}} \mathrm{H}^{*} \mathrm{PG} \mathbf{x}_{\mathrm{t}} \\
+\mathbf{u}_{\mathrm{t}}^{* \mathrm{op}} \mathrm{H}^{*} \mathrm{PH} \mathbf{u}_{\mathrm{t}}^{\text {op }}+\eta_{\mathrm{t}+1}^{*} \mathrm{P} \eta_{\mathrm{t}+1}>-\mathbf{x}_{\mathrm{t}}^{*} \mathrm{P} \mathbf{x}_{\mathrm{t}},(26)
\end{gathered}
$$

where we made use of the fact that the expected value of the random variable $\eta$ is zero. Now using (13) in (26) yields,

$$
\begin{aligned}
& \triangle \mathrm{V}(\mathbf{x}(\mathrm{t}))=<\mathbf{x}_{\mathrm{t}}^{*} \mathrm{G}^{*} \mathrm{PG} \mathbf{x}_{\mathrm{t}}+\mathbf{x}_{\mathrm{t}}^{*} \mathrm{G}^{*} \mathrm{PH} \mathrm{u}_{\mathrm{t}}^{\mathrm{op}} \\
& +\mathbf{u}_{\mathrm{t}}^{* \mathrm{op}} \mathrm{H}^{*} \mathrm{PG} \mathbf{x}_{\mathrm{t}}+\mathbf{u}_{\mathrm{t}}^{* \mathrm{op}} \mathrm{H}^{*} \mathrm{PH} u_{\mathrm{t}}^{o p}+\mathbf{x}_{\mathrm{t}}^{*} \mathrm{D}^{*} \mathrm{PD} \mathbf{x}_{\mathrm{t}} \\
& +\mathbf{x}_{\mathrm{t}}^{*} \mathrm{D}^{*} \mathrm{PE} \mathbf{u}_{\mathrm{t}}^{\mathrm{op}}+\mathbf{u}_{\mathrm{t}}^{* \mathrm{op}} \mathrm{E}^{*} \mathrm{PD} \mathbf{x}_{\mathrm{t}}+\mathbf{u}_{\mathrm{t}}^{* \mathrm{op}} \mathrm{E}^{*} \mathrm{PE} \mathbf{u}_{\mathrm{t}}> \\
& -<\mathbf{x}_{\mathrm{t}}^{*} \mathrm{P} \mathbf{x}_{\mathrm{t}}>\text {. }
\end{aligned}
$$

By referring to (17) and evaluating expectation of (27), it can be modified to

$$
\begin{gathered}
\triangle V(\mathbf{x}(\mathrm{t}))=\mathbf{x}_{\mathrm{t}}^{*} \mathrm{G}^{*} \mathrm{PG} \mathbf{x}_{\mathrm{t}}-\mathbf{x}_{\mathrm{t}}^{*} \mathrm{G}^{*} \mathrm{PHK} \mathrm{K}_{\mathrm{t}} \mathbf{x}_{\mathrm{t}} \\
-\mathbf{x}_{\mathrm{t}}^{*} \mathrm{~K}_{\mathrm{t}}^{*} \mathrm{H}^{*} \mathrm{PG} \mathbf{x}_{\mathrm{t}}+\mathbf{x}^{*} \mathrm{~K}_{\mathrm{t}}^{*} \mathrm{H}^{*} \mathrm{PHK} \mathrm{K}_{\mathrm{t}} \mathbf{x}_{\mathrm{t}}+\mathbf{x}_{\mathrm{t}}^{*}<\mathrm{D}^{*} \mathrm{PD}>\mathbf{x}_{\mathrm{t}} \\
-\mathbf{x}_{\mathrm{t}}^{*}<\mathrm{D}^{*} \mathrm{PE}>\mathrm{K}_{\mathrm{t}} \mathbf{x}_{\mathrm{t}}-\mathbf{x}_{\mathrm{t}}^{*} \mathrm{~K}_{\mathrm{t}}^{*}<\mathrm{E}^{*} \mathrm{PD}>\mathbf{x}_{\mathrm{t}} \\
+\mathbf{x}_{\mathrm{t}}^{*} \mathrm{~K}_{\mathrm{t}}^{*}<\mathrm{E}^{*} \mathrm{PE}>\mathrm{K}_{\mathrm{t}} \mathbf{x}_{\mathrm{t}}-\mathbf{x}_{\mathrm{t}}^{*} \mathrm{P} \mathbf{x}_{\mathrm{t}} .
\end{gathered}
$$

Equation (28) can be further modified to

$$
\begin{array}{r}
\triangle V(\mathbf{x}(\mathrm{t}))=\mathbf{x}_{\mathrm{t}}^{*}\left(\mathrm{G}-\mathrm{HK}_{\mathrm{t}}\right)^{*} \mathrm{P}\left(\mathrm{G}-\mathrm{HK}_{\mathrm{t}}\right) \mathbf{x}_{\mathrm{t}} \\
\left.+<\mathbf{x}_{\mathrm{t}}^{*}(\mathrm{D}-\mathrm{EK})_{\mathrm{t}}\right)^{*} \mathrm{P}\left(\mathrm{D}-\mathrm{EK} \mathrm{K}_{\mathrm{t}}\right) \mathbf{x}_{\mathrm{t}}>-\mathbf{x}_{\mathrm{t}}^{*} \mathrm{P} \mathbf{x}_{\mathrm{t}} .
\end{array}
$$

Since $V(\mathbf{x}(t))$ is chosen to be positive definite, it is required for asymptotic stability that $\triangle V(\mathbf{x}(t))$ be negative definite. Therefore,

$$
\begin{gathered}
\Delta V(\mathbf{x}(\mathrm{t}))=-\left(\mathrm{Q}+\mathrm{K}_{\mathrm{t}}^{*} R K_{\mathrm{t}}\right) \\
\left(\mathrm{G}-\mathrm{HK} \mathrm{K}_{\mathrm{t}}\right)^{*} \mathrm{P}\left(\mathrm{G}-\mathrm{HK}_{\mathrm{t}}\right) \\
+<\left(\mathrm{D}-\mathrm{EK} \mathrm{K}_{\mathrm{t}}\right)^{*} \mathrm{P}\left(\mathrm{D}-\mathrm{EK} \mathrm{K}_{\mathrm{t}}\right)>-\mathrm{P} \\
=-\left(\mathrm{Q}+\mathrm{K}_{\mathrm{t}}^{*} R \mathrm{~K}_{\mathrm{t}}\right) .
\end{gathered}
$$

Rearranging (30) so that it reads as,

$$
\begin{aligned}
& \mathrm{Q}+\mathrm{G}^{*} \mathrm{PG}-\mathrm{P}+\mathrm{K}_{\mathrm{t}}^{*}\left(\mathrm{R}+\mathrm{H}^{*} \mathrm{PH}+<\mathrm{E}^{*} \mathrm{PE}>\right) \mathrm{K}_{\mathrm{t}} \\
& -\mathrm{K}_{\mathrm{t}}^{*}\left(\mathrm{H}^{*} \mathrm{PG}+<\mathrm{E}^{*} \mathrm{PD}>\right)+<\mathrm{D}^{*} \mathrm{PD}> \\
& -\left(\mathrm{G}^{*} \mathrm{PH}+<\mathrm{D}^{*} \mathrm{PE}>\right) \mathrm{K}_{\mathrm{t}}=0 .
\end{aligned}
$$

By introducing the following definitions

$$
\begin{aligned}
\mathrm{M} & =\mathrm{H}^{*} \mathrm{PG}+<\mathrm{E}^{*} \mathrm{PD}>, \\
\mathrm{F} & =\mathrm{R}+\mathrm{H}^{*} \mathrm{PH}+<\mathrm{E}^{*} \mathrm{PE}>,
\end{aligned}
$$

equation (31) can be written as follows

$$
\begin{aligned}
& \quad \mathrm{Q}+\mathrm{G}^{*} \mathrm{PG}-\mathrm{P}+\mathrm{K}_{\mathrm{t}}^{*} \mathrm{FK}_{\mathrm{t}}-\mathrm{K}_{\mathrm{t}}^{*} \mathrm{M}-\mathrm{M}^{*} \mathrm{~K}_{\mathrm{t}} \\
& +<\mathrm{D}{ }^{*} \mathrm{PD}>=0 .
\end{aligned}
$$


This last equation can be modified as follows

$$
\begin{gathered}
\mathrm{Q}+\mathrm{G}^{*} \mathrm{PG}-\mathrm{P}+\left[\mathrm{F}^{1 / 2} \mathrm{~K}_{\mathrm{t}}-\mathrm{F}^{-1 / 2} \mathrm{M}\right]^{*} \\
\times\left[\mathrm{F}^{1 / 2} \mathrm{~K}_{\mathrm{t}}-\mathrm{F}^{-1 / 2} \mathrm{M}\right]-\mathrm{M}^{*} \mathrm{~F}^{-1} \mathrm{M}+<\mathrm{D}^{*} \mathrm{PD}>
\end{gathered}
$$$$
=0 \text {. }
$$

Minimization of $J$ with respect to $K_{t}$ requires minimization of the left hand side of equation (34) with respect to $K_{t}$. Since

$$
\left[\mathrm{F}^{1 / 2} \mathrm{~K}_{\mathrm{t}}-\mathrm{F}^{-1 / 2} \mathrm{M}\right]^{*}\left[\mathrm{~F}^{1 / 2} \mathrm{~K}_{\mathrm{t}}-\mathrm{F}^{-1 / 2} \mathrm{M}\right]
$$

is nonnegative, the minimum occurs when it is zero, or when

$$
\mathrm{F}^{1 / 2} \mathrm{~K}_{\mathrm{t}}=\mathrm{F}^{-1 / 2} \mathrm{M}
$$

Hence, we obtain

$$
\begin{aligned}
\mathrm{K}_{\mathrm{t}} & =\mathrm{F}^{-1} \mathrm{M} \\
& =\left[\mathrm{R}+\mathrm{H}^{*} \mathrm{PH}+<\mathrm{E}^{*} \mathrm{PE}>\right]^{-1} \\
& \times\left[\mathrm{H}^{*} \mathrm{PG}+<\mathrm{E}^{*} \mathrm{PD}>\right],
\end{aligned}
$$

where we made use of equations (32). Substitution of equation (37) into equation (34) gives

$$
\begin{aligned}
\mathrm{P} & =\mathrm{Q}+\mathrm{G}^{*} \mathrm{PG}+<\mathrm{D}^{*} \mathrm{PD}> \\
& -\left[\mathrm{G}^{*} \mathrm{PH}+<\mathrm{D}^{*} \mathrm{PE}>\right]\left[\mathrm{R}+\mathrm{H}^{*} \mathrm{PH}+<\mathrm{E}^{*} \mathrm{PE}>\right]^{-1} \\
& \times\left[\mathrm{H}^{*} \mathrm{PG}+<\mathrm{E}^{*} \mathrm{PD}>\right] .
\end{aligned}
$$

\section{References}

[1] Hossam Seddik Abbas and Herbert Werner. Frequencyweighted discrete-time lpv model reduction using structurally balanced truncation. IEEE Transactions on Control Systems Technology, 19(1):140-147, 2011.

[2] K. J. Åström and B. Wittenmark. On self tuning regulators. Automatica, 9:185-199, 1973.

[3] Peter. Benner, Jing-Rebecca. Li, and Thilo Penzl. Numerical solution of large-scale lyapunov equations, riccati equations, and linear-quadratic optimal control problems. Numerical Linear Algebra with Applications, 15:755-777, 2008.

[4] D. S. Bernstein and W. M. Haddad. Robust stability and performance via fixed order dynamic compensation with guaranteed cost bounds. Mathematics of Control, Signals and Systems, 3:139-163, 1990.

[5] R. L. Carroll and Mahgoub Hassan. An identification procedure for 2-D linear, time-invariant, discrete, systems using the direct method of lyapunov. In American Control Conference, pages 1137-1142, Minneapolis, MN, USA, June 1987.
[6] S. S. L. Chang and T. K. C. Peng. Adaptive guarnteed cost control of systems with uncertain parameters. IEEE Transactions on Automatic Control, 17:474-483, 1972.

[7] D. W. Clarke and P. J. Gawthrop. Self tuning control. IEE Proceedings, 126:633-640, 1979.

[8] S. Fabri and V. Kadirkamanathan. Dual adaptive control of nonlinear stochastic systems using neural networks. Automatica, 34(2):245-253, February 1998.

[9] S. G. Fabri and V. Kadirkamanathan. Functional Adaptive Control: An Intelligent Systems Approach. Springer-Verlag, February 2001.

[10] Hu Gang and Qu Zhilin. Controlling spatiotemporal chaos in coupled map lattice systems. Physical Review Letters, 72(1):68-73, January 1994.

[11] A. Gersho and R. M. Gray. Vector Quantization and Signal Compression. Kluwer Academic Publishers, 1992.

[12] G. C. Goodwin, P. J. Caines, and P. E. Caines. Discrete time stochastic adaptive control. SIAM Journal on Control and $\mathrm{Op}$ timization, 19(6):829-853, 1981.

[13] R. O. Grigoriev, M. C. Cross, and H. G. Schuster. Pinning control of spatiotemporal chaos. Physical Review Letters, 79(15):2795-2798, October 1997.

[14] S. Gugercin and A. Antoulas. A survey of model reduction by balanced truncation and some new results. International Journal of Control, 77(8):748-766, 2004.

[15] R. Herzallah. Probabilistic dual heuristic programming based adaptive critic. International Journal of Systems Science, 41:227-239, 2009.

[16] R. Herzallah. Non standard Ricatti solution and linear quadratic pinning control. In 19th Mediterranean Conference on Control and Automation, volume 1, pages 1504-1509, Corfu, Greece, June 2011.

[17] R. Herzallah and D. Lowe. A Bayesian perspective on stochastic neuro control. IEEE Transactions on Neural Networks, 19:1-11, April 2008.

[18] R. Herzallah and D. Lowe. Comparison between conventional and stochastic pinning control. In 19th Mediterranean Conference on Control and Automation, volume 1, pages 730-735, Corfu, Greece, June 2011.

[19] Randa. Herzallah and Miroslav. Káarnáy. Fully probabilistic control design in an adaptive critic framework. Neural Networks, 24(11):1128-1135, 2011.

[20] K. Kaneko. Period-doubling of kink-antikink patterns, quasiperiodicity in antiferro-like structures and spatial intermittency in coupled map logistic lattice - towards a prelude to a "field theory of chaos"-_." Progress of Theoretical Physics, 72(3):480-486, September 1984.

[21] N. Koshiya and J. C. Smith. Neuronal pacemaker for breathing visualized in vitro. Nature, 400:360-363, 1999.

[22] O. I. Kosmidou. Generalized riccati equations associated with guaranteed cost control: An overview of solutions and features. Applied Mathematics and Computation, 191:511-520, 2007.

[23] A. E. Motter, C. Zhou, and J. Kurths. Network synchronization, diffusion, and the paradox of heterogeneity. Physical Review E, 71:016116-1-016116-9, 2005.

[24] M. Ait. Rami, J. B. Moore, and Xun Yu Zhou. Indefinite stochastic linear quadratic control and generalized differential riccati equation. SIAM Journal on Control and Optimization, 40(4):1296-1311, 2001.

[25] Mustapha Ait Rami, Xi Chen, John B. Moore, and Xun Yu Zhou. Solvability and asymptotic behavior of generalized ric- 
cati equations arising in indefinite stochastic LQ control. IEEE Transactions on Automatic Control, 46(3):428-440, 2001.

[26] Mustapha Ait Rami and Laurent El Ghaoui. LMI optimization for nonstandard riccati equations arising in stochastic control. IEEE Transactions on Automatic Control, 41(11):1666-1671, 1996.

[27] F. Sorrentino, M. d. Bernardo, F. Garofalo, and G. Chen. Controllability of complex networks via pinning. Physical Review E, 75:046103-1-046103-6, April 2007.

[28] X. F. Wang and G. Chen. Pinning control of scale free dynamical networks. Physica A, 310:521-531, 2002.

[29] X. F. Wang and G. Chen. Complex networks: small-world, scale-free and beyond. IEEE Circuits and Systems Magazine, 3(1):6-20, 2003.

[30] L. Y. Xiang, Z. X. Liu, Z. Q. Chen, F. Chen, and Z. Z. Yuan. Pinning control of complex dynamical networks with general topology. Physica A, 379:298-306, 2007.

[31] W. Yu, G. Chen, and J. L. On pinning synchronization of complex dynamical networks. Automatica, 45:429-435, 2009.

[32] Xiang yu Gao Er-yong Cong Xian Zhang. Reliable linearquadratic optimal control for discrete-time singular linear systems. In Control and Decision Conference, 2009. CCDC'09. Chinese, pages 29-34, Guilin, June 2009. 